\title{
Endoplasmic reticulum stress regulates epithelial-mesenchymal transition in human lens epithelial cells
}

\author{
SHENG ZHOU*, JING YANG* ${ }^{*}$ MINGWEI WANG, DANYING ZHENG and YIZHI LIU \\ State Key Laboratory of Ophthalmology, Zhongshan Ophthalmic Center, \\ Sun Yat-sen University, Guangzhou, Guangdong 510060, P.R. China
}

Received May 8, 2019; Accepted October 24, 2019

DOI: $10.3892 / \mathrm{mmr} .2019 .10814$

\begin{abstract}
Epithelial-to-mesenchymal transition (EMT) of human lens epithelial cells (HLECs) serve an important role in cataract formation. The endoplasmic reticulum stress response (ER stress) has been demonstrated to regulate EMT in a number of tissues. The aim of the present study was to demonstrate the role of ER stress on EMT in HLECs. HLECs were treated with tunicamycin (TM) or thapsigargin (TG) to disturb ER homeostasis, and 4-phenylbutyric acid (PBA) or sodium tauroursodeoxycholate (TUDCA) to restore ER homeostasis. Cell morphology was evaluated after $24 \mathrm{~h}$. The long axis and aspect ratio of the cells were analyzed using ImageJ software. The results demonstrated that HLECs adopted an elongated morphology following treatment with $\mathrm{TG}$, and the cellular aspect ratio increased. However, this morphological change was not observed following combination treatment with TG and PBA. Western blot analysis and immunofluorescence staining were used to measure the protein expression levels. A wound-healing assay was performed to evaluate cell migration. Treatment with TM
\end{abstract}

Correspondence to: Professor Yizhi Liu, State Key Laboratory of Ophthalmology, Zhongshan Ophthalmic Center, Sun Yat-sen University, 54S Xianlie, Guangzhou, Guangdong 510060, P.R. China E-mail: yizhi_liu@aliyun.com

*Contributed equally

Abbreviations: HLECs, human lens epithelial cells; ER stress, endoplasmic reticulum stress response; ER, endoplasmic reticulum; EMT, epithelial-to-mesenchymal transition; PCO, posterior capsular opacification; UPR, unfolded protein response; $\alpha$-SMA, $\alpha$-smooth muscle actin; GRP78, glucose-regulated protein 78; IRE1 $\alpha$, phosphorylated inositol-requiring protein $1 \alpha$; eIf $2 \alpha$, phosphorylated eukaryotic initiation factor $2 \alpha$; -, phosphorylated; ATF6, activating transcription factor 6; ATF4, activating transcription factor 4; TG, thapsigargin; TM, tunicamycin; PBA, 4-phenylbutyric acid; TUDCA, tauroursodeoxycholate

Key words: human lens epithelial cells, endoplasmic reticulum stress response, epithelial-to-mesenchymal transition, unfolded protein response or TG increased the expression of the ER stress markers glucose-regulated protein 78, phosphorylated eukaryotic initiation factor $2 \alpha$, activating transcription factor (ATF)6, ATF4 and inositol-requiring protein $1 \alpha$ and the EMT markers fibronectin, vimentin, $\alpha$-smooth muscle actin and neural cadherin. Furthermore, treatment with TM or TG decreased the expression of the epithelial cell marker epithelial cadherin and enhanced cell migration, which effects were inhibited following treatment with PBA or TUDCA. These results indicates that enhanced ER stress induced EMT and subsequently increased cell migration in HLECs in vitro.

\section{Introduction}

Cataracts are generally treated surgically; however, excess proliferation and differentiation of the remaining human lens epithelial cells (HLECs) may result in vision disturbance following surgery (1-3). Epithelial-to-mesenchymal transition (EMT) has been implicated in the transition of HLECs to myofibroblasts $(4,5)$.

EMT cell characteristics include the acquisition of a spindle-shaped morphology that is accompanied by an accumulation of $\alpha$-smooth muscle actin ( $\alpha$-SMA), a redistribution of actin stress fibers, a loss of cell polarity and epithelial markers such as cytokeratin, zonula occludens-1 and epithelial cadherin (E-cadherin), and upregulation of transcription factors including snail family transcriptional repressor 1 and 2 and twist family bHLH transcription factor 1 (6-11).

Previous studies have revealed that cataract surgery may result in cellular stress $(12,13)$. The endoplasmic reticulum (ER) serves an important role in detecting cellular stress, and subsequently triggers the ER stress response (ER stress) to restore cellular homeostasis. Additionally, the unfolded protein response (UPR) is triggered alongside ER stress to additionally decrease cellular stress (14).

Evidence indicates that the UPR participates in crosstalk with EMT in several types of cells: The UPR potentiates EMT in gastric cancer cells under conditions of severe hypoxia (15) or prolonged ER stress, and results in irreversible EMT in human peritoneal mesothelial cells (16). However, whether ER stress affects EMT in the human lens epithelium remains unclear. Therefore, the present study evaluated the role of ER stress in inducing EMT in HLECs. ER stress resulted in morphological changes, increased cell migration and altered 
expression of EMT-associated proteins in a human lens epithelial cell line in vitro. Together, these results suggested that ER stress serves an important role in regulating EMT in HLECs.

\section{Materials and methods}

Reagents and antibodies. The ER stress activators thapsigargin (TG) and tunicamycin (TM) were purchased from Sigma-Aldrich; Merck KGaA and Beijing Solarbio Science \& Technology Co., Ltd., respectively. The ER stress inhibitors 4-phenylbutyric acid (PBA) and sodium tauroursodeoxycholate (TUDCA) were purchased from Sigma-Aldrich; Merck KGaA. TM, TG, PBA and TUDCA were dissolved in dimethyl sulfoxide (DMSO; Leagene). Anti-glucose-regulated protein $78 \mathrm{kDa}$ (GRP78; ab12223), anti-activating transcription factor (ATF)6 (ab11909), anti-phosphorylated eukaryotic initiation factor $2 \alpha$ (p-IRE1 $\alpha$; ab48187), anti-E-Cadherin (ab40772), anti-fibronectin (ab2413) and anti- $\alpha$-SMA (ab32575) primary antibodies were purchased from Abcam. Horseradish peroxidase-conjugated anti-p-eIF2 $\alpha$ (119A11), horse anti-mouse and horse anti-rabbit secondary antibodies, Alexa Fluor 488-conjugated goat anti-rabbit and Alexa Fluor 488-conjugated goat anti-mouse secondary antibodies were purchased from Cell Signaling Technology, Inc. Anti-ATF4 primary antibody (sc-390063) was purchased from Santa Cruz Biotechnology, Inc. Primary antibodies against vimentin (10366-1-AP), $\beta$-actin (66009-1-Ig) and Neural cadherin (N-cadherin; 22018-1-AP) were purchased from ProteinTech Group, Inc.

HLEC culture and treatment. The human lens epithelial SRA01/04 cell line (supplied by Professor Shang, Zhongshan Ophthalmic Center) was cultured in Dulbecco's modified Eagle's medium (Gibco; Thermo Fisher Scientific, Inc.) supplemented with $10 \%$ fetal bovine serum (Gibco; Thermo Fisher Scientific, Inc.) and $1 \%$ penicillin/streptomycin at $37^{\circ} \mathrm{C}$. In order to evaluate the role of ER stress, SRA01/04 cells were treated with TM, TG, PBA and TUDCA for $24 \mathrm{~h}$ at $37^{\circ} \mathrm{C}$ at the concentrations listed below.

HLEC morphological analysis. SRA01/04 cells were treated with $0.01 \mu \mathrm{M}$ TG or a combination of $0.01 \mu \mathrm{M} \mathrm{TG}$ and $0.25 \mathrm{mM}$ PBA for $24 \mathrm{~h}$. Untreated SRA01/04 cells served as the control group. Cell morphology was analyzed under an inverted phase-contrast microscope (Axiovert 200; Carl Zeiss AG), and images were captured using a digital camera (AxioCam HRC; Carl Zeiss AG; magnification x20). A minimum of 9 images per group were analyzed using ImageJ software 1.8.0 (National Institutes of Health) and the length of the long axis of the cells and the aspect ratio, defined as the ratio of the long axis (width) to the short axis (length) of the cells, were determined. The experiment was performed in triplicate.

Western blot analysis. SRA01/04 cells were treated with $0.01 \mu \mathrm{M}$ TG, $0.01 \mu \mathrm{M}$ TG and $0.25 \mathrm{mM}$ PBA, $0.01 \mu \mathrm{M}$ TG and $2 \mathrm{mM}$ TUDCA, $0.1 \mu \mathrm{M}$ TM, $0.1 \mu \mathrm{M}$ TM and $0.25 \mathrm{mM}$ PBA or $0.1 \mu \mathrm{M}$ TM and $2 \mathrm{mM}$ TUDCA for $24 \mathrm{~h}$. Untreated and DMSO-treated SRA01/04 cells served as the control groups. Total protein was subsequently extracted from the cells using radioimmunoprecipitation assay buffer. Protein samples (30 $\mu \mathrm{g}$ protein/lane) were separated via SDS-PAGE on a $12 \%$ gel. The separated proteins were subsequently transferred onto a polyvinylidene fluoride membrane and blocked for 90 min with 5\% skim milk in TBST (0.05\% Tween) at room temperature. The membrane was incubated with primary antibodies against GRP78, ATF6, ATF4, P-eIF2 $\alpha$, P-IRE1 $\alpha$, fibronectin, vimentin, $\alpha$-SMA, N-cadherin, E-cadherin and $\beta$-actin (all 1:1,000) overnight at $4^{\circ} \mathrm{C}$. Following the primary incubation, membranes were incubated with horseradish peroxidase-conjugated horse anti-mouse or horse anti-rabbit secondary antibodies at room temperature for $90 \mathrm{~min}$. The membrane was then incubated with enhanced chemiluminescence substrates (ECL-WBKLS0100; EMD Millipore) at room temperature for $2 \mathrm{~min}$ and the protein bands were visualized using a ChemiDoc XRS+ imager. Protein expression was quantified using Image Lab software (Imagelab 4.0) with $\beta$-actin as the loading control.

Immunofluorescence analysis. SR A01/04 cells were treated as aforementioned in the previous paragraph (western blot analysis) and $4 \times 10^{5}$ cells were seeded onto cover slips in a 12 -well plate and cultured for $24 \mathrm{~h}$. Cells were subsequently fixed with ice-cold acetone for $15 \mathrm{~min}$, permeabilized with $0.5 \%$ Triton X-100 for $5 \mathrm{~min}$ and blocked with $1 \%$ bovine serum albumin in PBS for $90 \mathrm{~min}$ at room temperature. The cells were incubated with primary antibodies against fibronectin, vimentin, $\alpha$-SMA and $\mathrm{N}$-cadherin (all 1:200) overnight at $4^{\circ} \mathrm{C}$. Subsequently, the cells were washed with PBS containing $0.1 \%$ Tween-20 at room temperature and then incubated with Alexa Fluor 488-conjugated goat anti-rabbit or Alexa Fluor 488-conjugated goat anti-mouse secondary antibodies $(1: 5,000)$ at room temperature for $90 \mathrm{~min}$. VECTASHIELD Mounting Medium with DAPI (H1200, Vector Laboratories, Inc.) was used for DAPI staining at room temperature. Cells were observed under a fluorescence microscope (AX70; Olympus Corporation; magnification, $\mathrm{x} 20$ ). The relative fluorescence intensity of the specific proteins was quantified in 5 randomly-selected fields using ImageJ software 1.8.0 (National Institutes of Health), and normalized to the total number of cells. Data from 3 images per group were used for statistical analysis.

Wound-healing assay. A wound-healing assay was performed to assess the migration ability of SRA01/04 cells under specific ER stress conditions. A serum starving method was used for minimizing cell proliferation in the wound-healing assay. Dulbecco's Modified Eagle Medium supplemented with $0.5 \%$ fetal bovine serum and $1 \%$ penicillin/streptomycin was used for culturing the cells at $37^{\circ} \mathrm{C}$. The cells were treated with $0.01 \mu \mathrm{M} \mathrm{TG}, 0.1 \mu \mathrm{M} \mathrm{TM}, 0.01 \mu \mathrm{M}$ TG and $0.25 \mathrm{mM}$ or $0.1 \mu \mathrm{M}$ TM and $0.25 \mathrm{mM}$ PBA for $24 \mathrm{~h}$. Untreated and DMSO-treated SRA01/04 cells served as the control groups. Sterilized pipette tips were used to create a wound across the confluent cell monolayer, and debris was removed with PBS. Migration of the cells into the wound was observed after $24 \mathrm{~h}$ in 5 randomly-selected fields under an inverted phase-contrast microscope at magnification, $\mathrm{x} 40$. Cell migration was analyzed using ImageJ software (National Institutes of Health) and was expressed as the repair rate of scarification, defined as the percentage of the gap relative to the total area of the cell-free region immediately following wounding. Experiments were performed in triplicate at least 3 times. 
Statistical analysis. The results are expressed as the mean \pm standard deviation of at least 3 independent experiments. Statistical analysis was performed using GraphPad Prism software (v7; GraphPad Software, Inc.). A one-way analysis of variance followed by Tukey's post hoc test was used to compare the different groups. $\mathrm{P}<0.05$ was considered to indicate a statistically significant difference.

\section{Results}

HLEC morphology is altered following prolonged ER stress. In order to investigate the effect of ER stress on cell morphology and alignment, SRA01/04 cells were treated with DMSO, TG and a combination of TG and PBA. Representative images of SRA01/04 cells from each treatment group are presented in Fig. 1A. Compared with the other groups, TG-treated cells had a spindle-like appearance and an elongated long axis (Fig. 1B). The results indicated that activation of ER stress by TG had a significant effect on SRA01/04 cell elongation, and that this effect was eliminated by the ER stress inhibitor PBA. The morphology of TG-treated cells resembled that of fiber cells rather than epithelial cells.

TM and TG trigger ER stress in HLECs. The present study revealed that TG altered the morphology of SRA01/04 cells and that this effect was inhibited by PBA. ER stress following treatment with TM, TG, PBA or TUDCA was subsequently investigated. Western blot analysis was performed to determine the levels of proteins associated with 3 pathways of the UPR. The expression levels of GRP78, p-eIf2 $\alpha$, ATF6, ATF4 and P-IRE1 $\alpha$ expression in SRA01/04 cells were significantly increased following treatment with TM or TG and significantly decreased following treatment PBA or TUDCA (Fig. 1C and D). The results suggested that ER stress was triggered by TM or TG and inhibited by PBA or TUDCA.

ER stress facilitates EMT in HLECs. To address the effect of ER stress on EMT, SRA01/04 cells were exposed to the classic ER stress inducers TM or TG alone or in combination with classic ER stress inhibitors PBA or TUDCA for $24 \mathrm{~h}$. The expression of fibronectin, vimentin, $\alpha$-SMA, N-cadherin, E-cadherin and $\beta$-actin was measured by western blot analysis. Compared with the control groups, N-cadherin, vimentin, $\alpha$-SMA and fibronectin protein levels in TM-treated cells were increased by 1.82-, 1.76-, 2.18- and 2.69-fold, respectively. Furthermore, the protein level of E-cadherin decreased to $37 \%$ of the control groups (Fig. 2A). Compared with the control groups, $\mathrm{N}$-cadherin, vimentin, $\alpha$-SMA and fibronectin protein levels in TG-treated cells were increased by 2.21-, 1.66-, 2.49- and 1.86-fold. Furthermore, the protein level of E-cadherin had decreased by 0.61 -fold (Fig. 2B). By contrast, SRA01/04 cells treated with a combination of PBA or TUDCA and TM or TG did not exhibit significant changes in the expression of the aforementioned EMT-associated proteins compared with the control groups (Fig. 2A and B). These results indicated that ER stress triggered EMT in SRA01/04 cells, and immunofluorescence staining was performed to corroborate these results. As expected, the average fluorescence intensities corresponding to fibronectin, vimentin, $\alpha$-SMA and $\mathrm{N}$-cadherin per cell were significantly increased in the TM- and TG-treated groups compared with the control groups (Fig. 3). Furthermore, the increased expression was attenuated in cells treated with a combination of TM and TUDCA or TG and TUDCA.

ER stress enhances migration of HLECs. The wound-healing assay revealed that ER stress promoted the migration of SRA01/04 cells (Fig. 4A and B). The migration of SRA01/04 cells was significantly increased in TM- or TG-treated cells compared with the untreated control group. The repair rate of scarification of the TM- and TG-treated cells after $24 \mathrm{~h}$ was 60 and $65 \%$, respectively. However, cells treated with a combination of TM and PBA or TG and PBA did not exhibit a significant difference in the repair rate of scarification compared with the control group. The DMSO-treated cells served as the negative control. No significant differences were observed between the DMSO-treated cells and the untreated cells.

\section{Discussion}

The present study investigated the role of ER stress on EMT in HLECs. ER stress triggers a response termed the UPR, which serves to decrease cell stress (17). The UPR is activated following cellular stress in several disorders, including cataracts (18). A previous study revealed that ER stress and the UPR participate in the formation of several types of cataracts (19). In the present study, TM and TG were used to activate the UPR, and PBA and TUDCA were used to inhibit its activation. TM- or TG-treated SRA01/04 cells exhibited increased GRP78, p-eIf2 $\alpha$, ATF6, ATF4 and P-IRE1 $\alpha$ expression levels compared with the control groups, indicating that 3 UPR pathways were activated. Conversely, treatment with PBA or TUDCA decreased the expression of these proteins. The results obtained in the present study are in concordance with the well-known effects of TM, TG, PBA and TUDCA on HLECs.

During EMT, epithelial cancer cells lose polarity and cell-cell adhesion while gaining a more mesenchymal and invasive phenotype. Additionally, the cells appear to adopt a fibroblastic spindle-like morphology $(7,20)$. The present study revealed that SRA01/04 cells exhibited a more spindle-like morphology following treatment with TG. Additionally, the long axis of the cells was significantly elongated compared with the control group.

The present study subsequently investigated whether chemically-induced ER stress may have an effect on EMT in SRA01/04 cells. Typical markers for EMT include the downregulation of E-cadherin and the upregulation of $\mathrm{N}$-cadherin, vimentin, fibronectin and $\alpha$-SMA (21). Previous studies revealed that ER stress and EMT markers are commonly observed under stress conditions in multiple tissues, including the lungs, liver and kidneys (22-26). The western blot analysis and immunofluorescence staining performed in the present study revealed a decreased expression level of E-cadherin and increased expression levels of $\mathrm{N}$-cadherin, vimentin, fibronectin and $\alpha$-SMA in TM- or TG-treated SRA01/04 cells compared with the controls. However, these changes in expression were not significant when the disruption of ER stress was inhibited by TUDCA or PBA, suggesting that ER stress may serve regulatory role in EMT. 
A

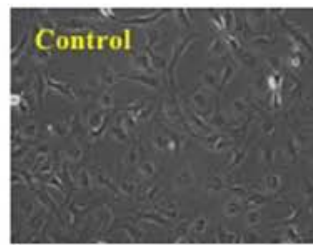

TG

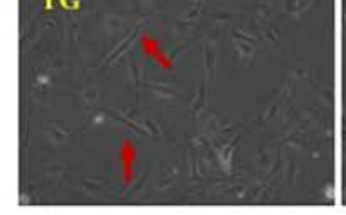

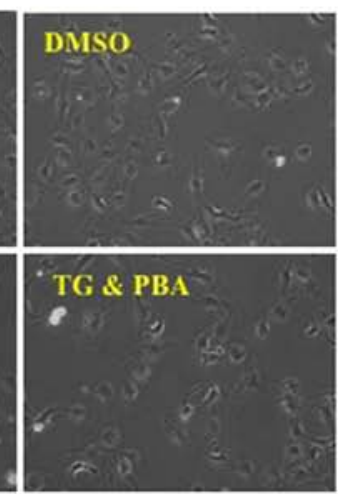

B

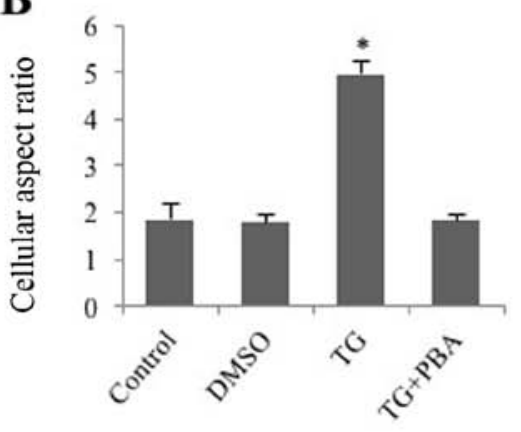

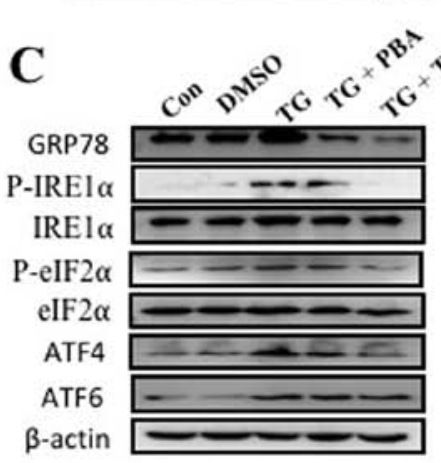

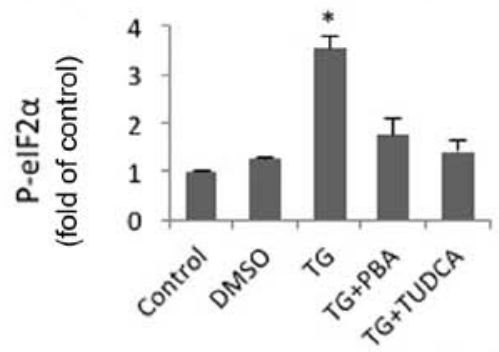

D
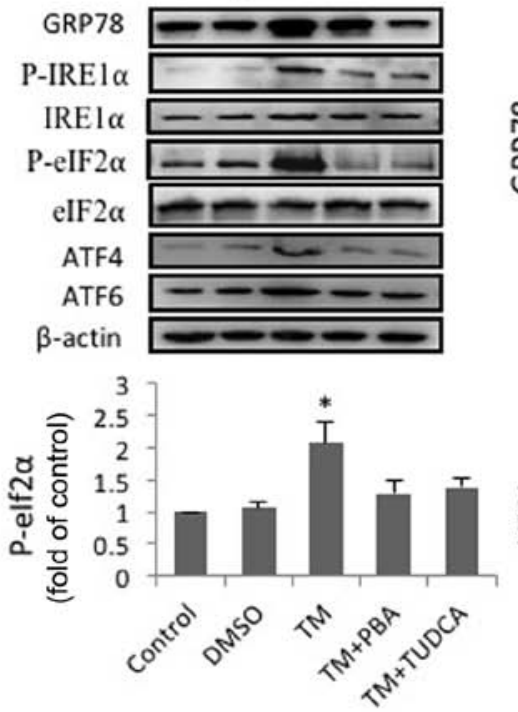
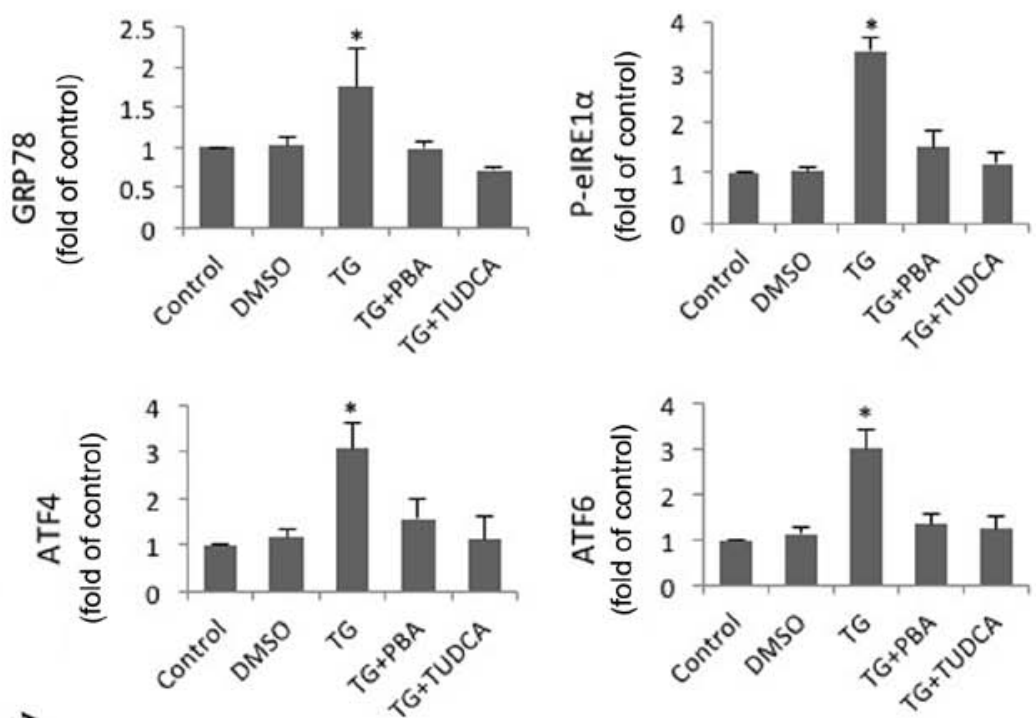
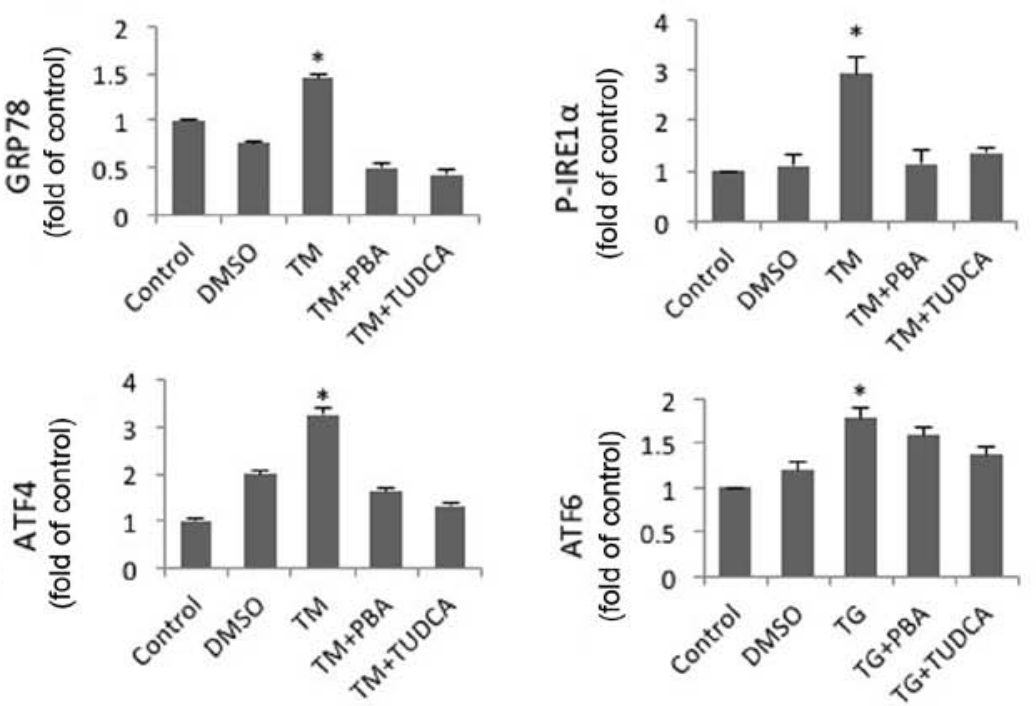

Figure 1. SRA01/04 cells treated with endoplasmic reticulum stress inducers exhibit a morphological change, from an epithelial- to a fibroblast-like morphology. SRA01/04 cells were treated with $0.01 \mu \mathrm{M}$ TG or a combination of $0.01 \mu \mathrm{M}$ TG and $0.25 \mathrm{mM}$ PBA for $24 \mathrm{~h}$. Untreated SRA01/04 cells served as the control group. (A) Micrographs were obtained under an inverted phase-contrast microscope (magnification, x20). The red arrows indicate that TG-treated cells had a spindle-like appearance and an elongated long axis. (B) The cellular aspect ratio was analyzed with ImageJ software (n=3). $\mathrm{P}<0.05 \mathrm{vs.} \mathrm{control.} \mathrm{SRA01/04} \mathrm{cells}$ were treated with $0.01 \mu \mathrm{M}$ TG, $0.01 \mu \mathrm{M}$ TG and $0.25 \mathrm{mM}$ PBA, $0.01 \mu \mathrm{M}$ TG and $2 \mathrm{mM}$ TUDCA, $0.1 \mu \mathrm{M}$ TM, $0.1 \mu \mathrm{M}$ TM and $0.25 \mathrm{mM}$ PBA or $0.1 \mu \mathrm{M}$ TM and $2 \mathrm{mM}$ TUDCA for $24 \mathrm{~h}$. (C) ER stress markers were determined by western blot analysis. (D) The expression levels of GRP78, p-IRE1 $\alpha$, p-eIF2 $\alpha$, ATF4 and ATF6 were quantified by densitometry $(n=3)$. * $\mathrm{P}<0.05$ vs. control. TG, thapsigargin; TM, tunicamycin; PBA, 4-phenylbutyric acid; TUDCA, tauroursodeoxycholate; GRP78, glucose-regulated protein $78 \mathrm{kDa}$; P-IRE1 $\alpha$, phosphorylated inositol-requiring protein $1 \alpha$; P-eIf $2 \alpha$, phosphorylated eukaryotic initiation factor $2 \alpha$; ATF6, activating transcription factor 6; ATF4, activating transcription factor 4; DMSO, dimethyl sulfoxide; Con, control. 
$\mathbf{A}$
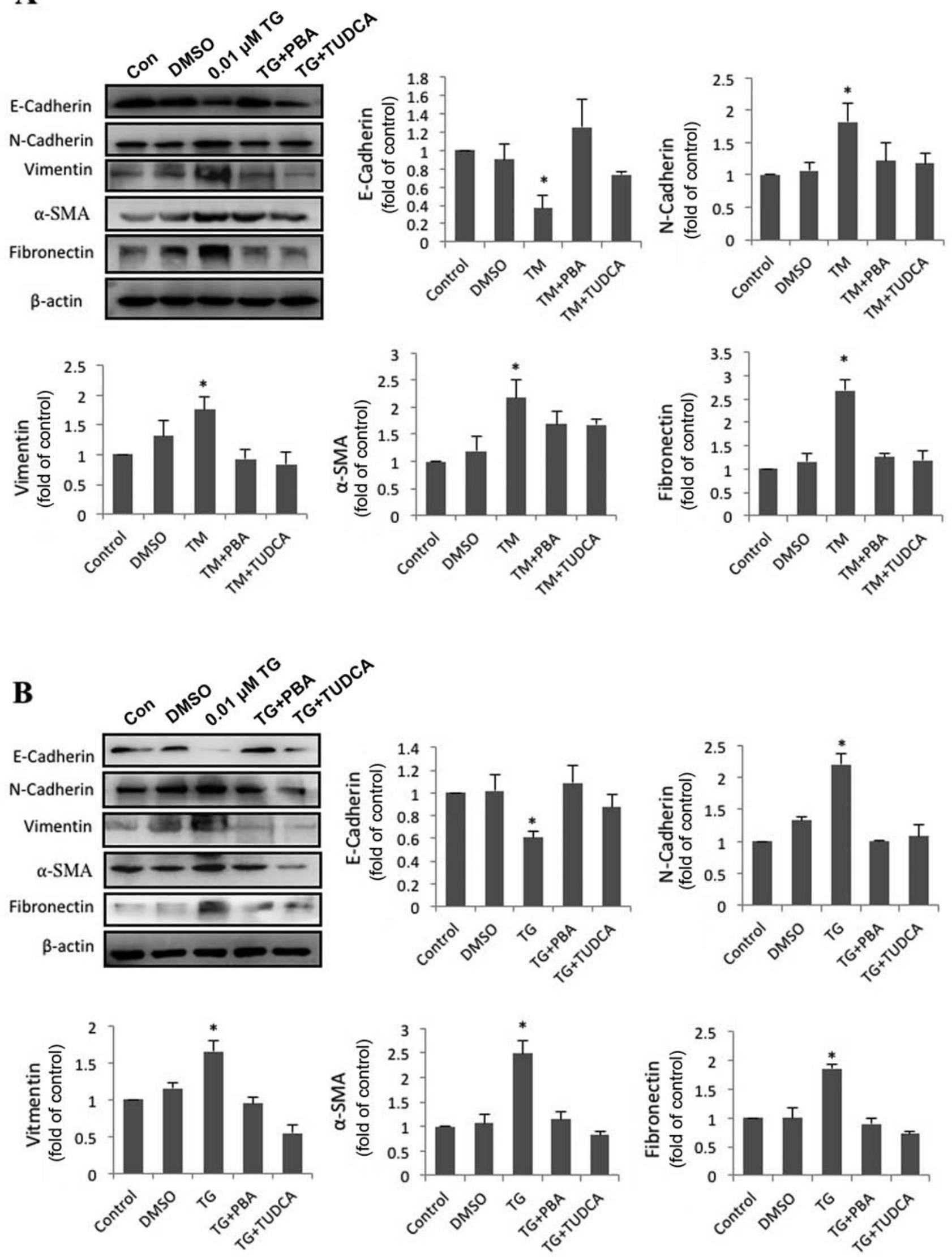

Figure 2. Enhanced endoplasmic reticulum stress upregulates EMT-associated protein expression and downregulates epithelial marker expression. (A) SRA01/04 cells were treated with $0.1 \mu \mathrm{M} \mathrm{TM}, 0.1 \mu \mathrm{M}$ TM and $0.25 \mathrm{mM}$ PBA or $0.1 \mu \mathrm{M}$ TM and $2 \mathrm{mM}$ TUDCA for $24 \mathrm{~h}$. (B) SRA01/04 cells were treated with $0.01 \mu \mathrm{M} \mathrm{TG}, 0.01 \mu \mathrm{M}$ TG and $0.25 \mathrm{mM}$ PBA or $0.01 \mu \mathrm{M}$ TG and $2 \mathrm{mM}$ TUDCA for $24 \mathrm{~h}$. The expression levels of the EMT-associated markers fibronectin, vimentin, $\alpha$-SMA, N-cadherin and E-cadherin were measured by western blot analysis $(n=3)$. ${ }^{*} \mathrm{P}<0.05$ vs. control. EMT, epithelial-to-mesenchymal transition; TG, thapsigargin; TM, tunicamycin; PBA, 4-phenylbutyric acid; TUDCA, tauroursodeoxycholate; $\alpha$-SMA, $\alpha$-smooth muscle actin. N-cadherin, neural cadherin; E-cadherin, epithelial cadherin; DMSO, dimethyl sulfoxide; Con, control. 


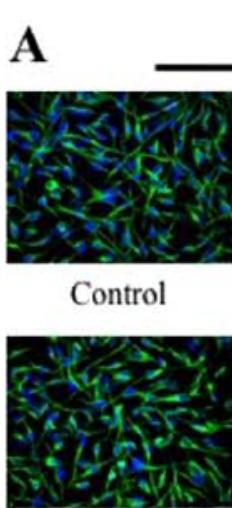

DMSO

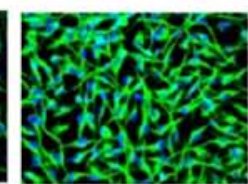

TM

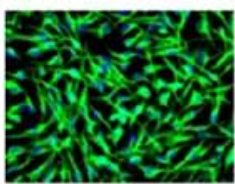

TG
Vimentin / DAPI

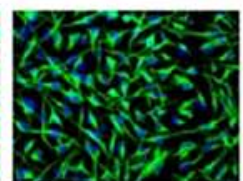

$\mathrm{TM}+\mathrm{PBA}$

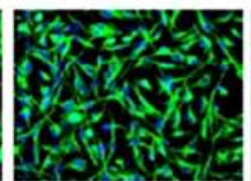

$\mathrm{TG}+\mathrm{PBA}$

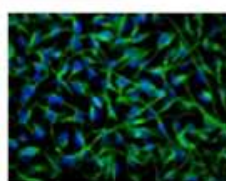

TM +TUDCA

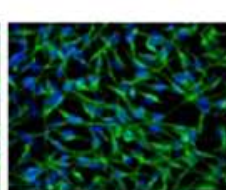

TG +TUDCA
C

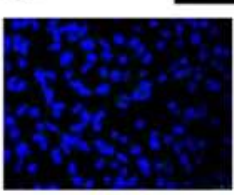

Control

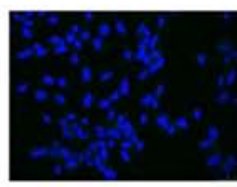

DMSO

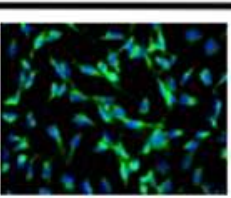

TM

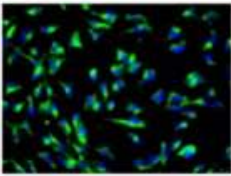

TG

$\mathrm{N}$-Cadherin / DAPI

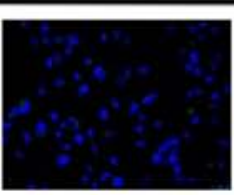

$\mathrm{TM}+\mathrm{PBA}$

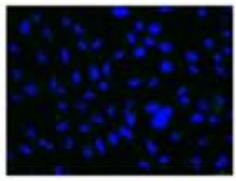

$\mathrm{TG}+\mathrm{PBA}$
B
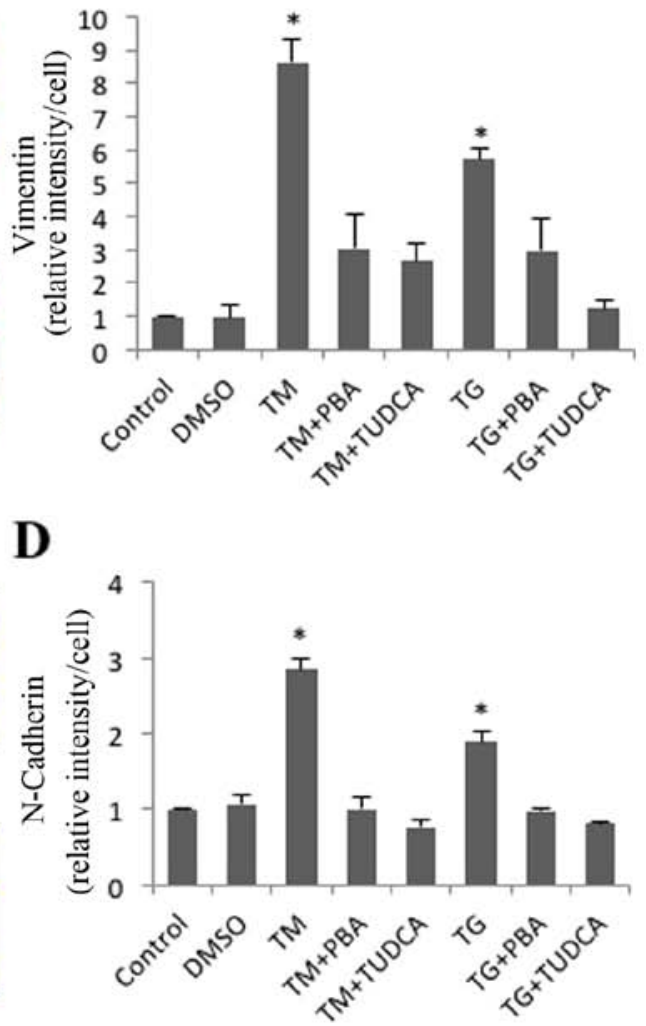

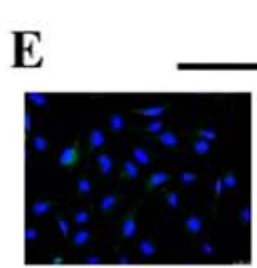

Control

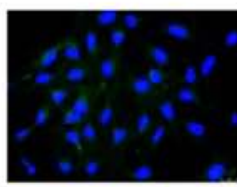

DMSO

$\alpha-S M A / D A P I$

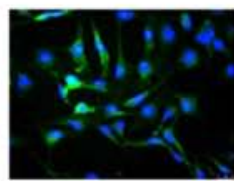

TM

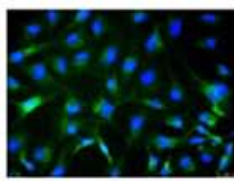

TG

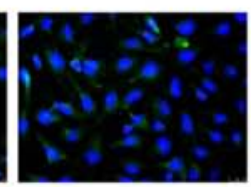

$\mathrm{TM}+\mathrm{PBA}$

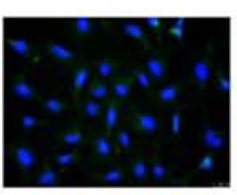

$\mathrm{TG}+\mathrm{PBA}$
D

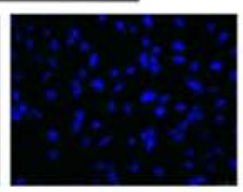

TM + TUDCA

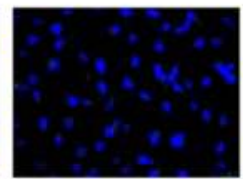

TG + TUDCA
D

F

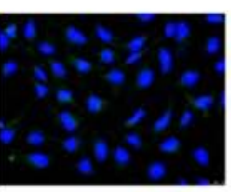

$\mathrm{TM}+\mathrm{TUDCA}$

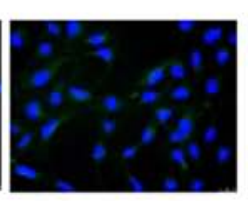

TG +TUDCA

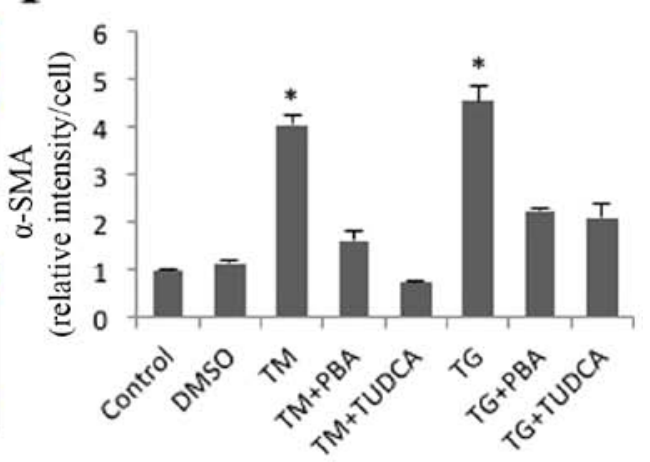

G

Fibronectin / DAPI

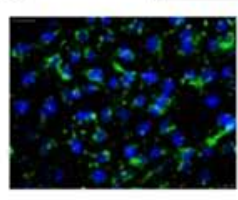

Control

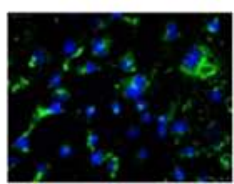

DMSO

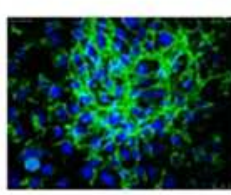

TM

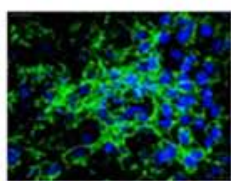

TG

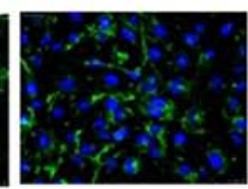

$\mathrm{TM}+\mathrm{PBA}$

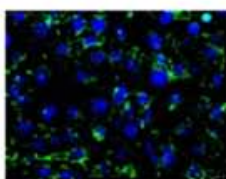

$\mathrm{TG}+\mathrm{PBA}$

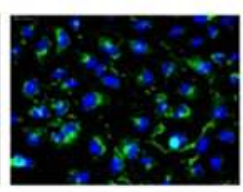

TM + TUDCA

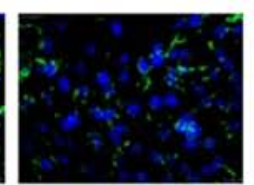

TG + TUDCA
H

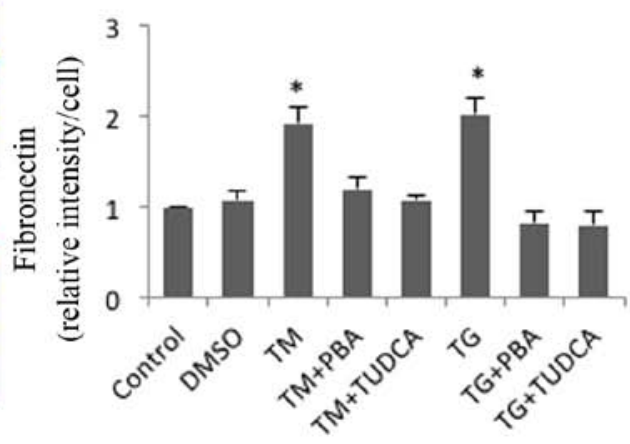

Figure 3. Immunofluorescence analysis of endoplasmic reticulum stress-induced expression of vimentin, N-cadherin, $\alpha$-SMA and fibronectin in SRA01/04 cells. SRA01/04 cells were treated with $0.01 \mu \mathrm{M} \mathrm{TG}, 0.01 \mu \mathrm{M}$ TG and $0.25 \mathrm{mM}$ PBA, $0.01 \mu \mathrm{M}$ TG and $2 \mathrm{mM}$ TUDCA, $0.1 \mu \mathrm{M}$ TM, $0.1 \mu \mathrm{M}$ TM and $0.25 \mathrm{mM}$ PBA or $0.1 \mu \mathrm{M}$ TM and $2 \mathrm{mM}$ TUDCA for $24 \mathrm{~h}$. The expression of (B) vimentin, (D) N-cadherin, (F) $\alpha-S M A$ and (H) fibronectin was determined by immunofluorescence analysis. The average fluorescence of (A) vimentin, (C) N-cadherin, (E) $\alpha$-SMA and (G) fibronectin was quantified ( $\mathrm{n}=3$ ). * $\mathrm{P}<0.05$ vs. control, magnification, x20. $\alpha$-SMA, $\alpha$-smooth muscle actin; N-cadherin, neural cadherin; E-cadherin; epithelial cadherin; TG, thapsigargin; TM, tunicamycin; PBA, 4-phenylbutyric acid; TUDCA, tauroursodeoxycholate. 
A
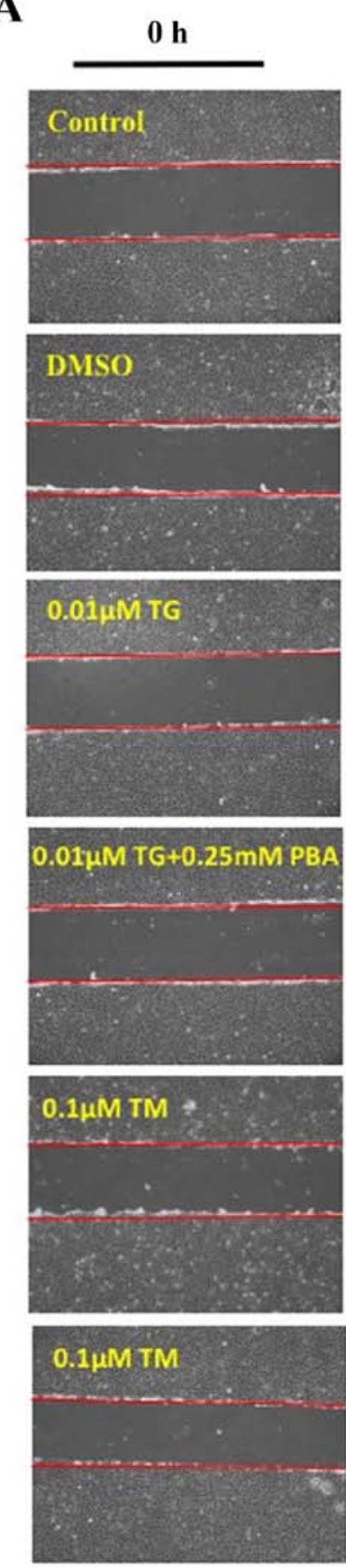

24 h
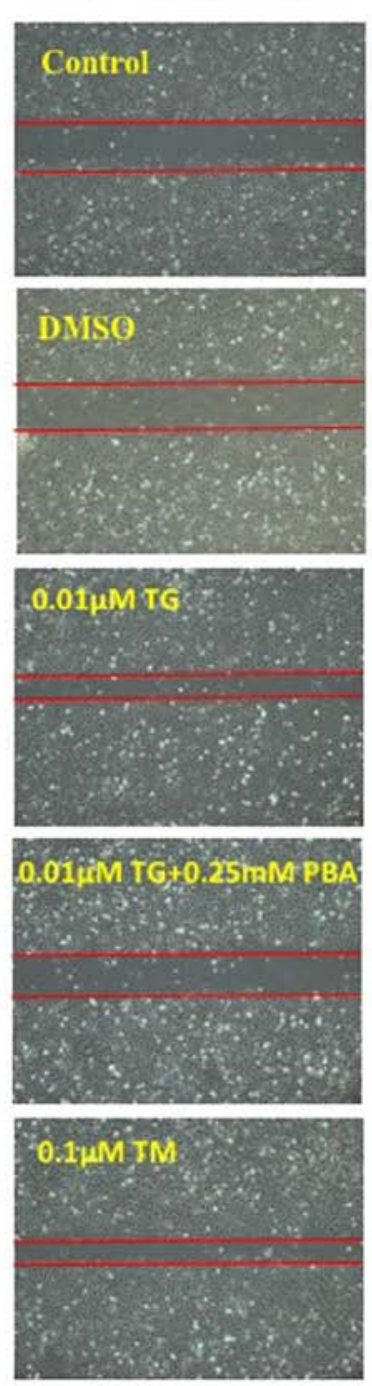

$0.1 \mu \mathrm{M} T \mathrm{TM}+0.25 \mathrm{miM} P \mathrm{PBA}$

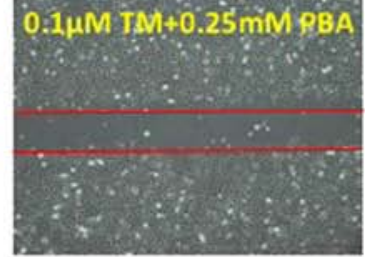

B

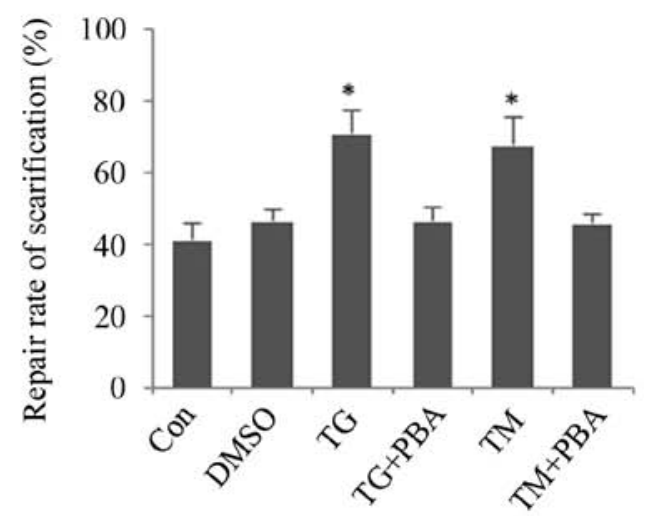

Figure 4. Endoplasmic reticulum stress facilitates the cell migration of SRA01/04 cells. SRA01/04 cells were treated with $0.01 \mu \mathrm{M} \mathrm{TG}, 0.1 \mu \mathrm{M}$ TM, $0.01 \mu \mathrm{M}$ TG and $0.25 \mathrm{mM}$ or $0.1 \mu \mathrm{M} \mathrm{TM}$ and $0.25 \mathrm{mM}$ PBA for $24 \mathrm{~h}$ and were subsequently subjected to a wound healing assay. Untreated and dimethyl sulfoxide-treated SRA01/04 cells served as the control groups. (A) Cells that migrated into the wounded area from the border of the wound after $24 \mathrm{~h}$ were visualized and images were captured under an inverted phase-contrast microscope (magnification, x10). (B) Cell migration was used to calculate the repair rate of scarification, expressed as the percentage of the gap relative to the total area of the cell-free region, using ImageJ software ( $\mathrm{n}=3$ ). ${ }^{*} \mathrm{P}<0.05$ vs. control. TG, thapsigargin; TM, tunicamycin; PBA, 4-phenylbutyric acid; TUDCA, tauroursodeoxycholate; $\alpha$-SMA, $\alpha$-smooth muscle actin; DMSO, dimethyl sulfoxide; Con, control.

Cataract surgery is an effective treatment strategy; however, it is not possible to completely remove the lens epithelial cells attached to an intact lens capsule. A number of previously published studies suggested that EMT is implicated in the pathogenesis of fibrotic posterior capsular opacification (PCO) $(27,28)$. Therefore, an increased understanding of how cataract surgery initiates EMT and how to modulate EMT following cataract surgery is required. The present study investigated the migration of SRA01/04 cells under conditions that regulate ER stress. The results revealed that TM- or TG-treated cells exhibited enhanced migration compared with the control groups, an effect that was abrogated in the presence of TUDCA or PBA.

In summary, the results from the present study suggested that ER stress is an important regulator of EMT in HLECs. Therefore, suppressing ER stress and regulating the EMT may serve as a novel therapeutic strategy to reduce PCO following cataract surgery. 


\section{Acknowledgements}

The authors would like to thank Professor Fu Shang (Zhongshan Ophthalmic Center) who provided the human lens epithelial cell line SRA01/04 for the present study.

\section{Funding}

The present study was supported by the National Natural Science Foundation of China (grant no. 81500742) awarded to JY, the Science and Technology Foundation of Guangdong Province of China (grant no. 2017A020215187) awarded to JY and the Natural Science Research Foundation of Guangdong Province of China (grant nos. 2017A030313614 and 2018A030313117) awarded to SZ.

\section{Availability of data and materials}

The datasets used and/or analyzed during the current study are available from the corresponding author on reasonable request.

\section{Authors' contributions}

SZ performed the western blot analysis, statistical analysis and participated in drafting the manuscript. JY performed the molecular experiments, participated in the design of the study, revised the statistical analysis and drafted the manuscript. MW performed the immunofluorescence staining and statistical analysis. DZ participated in designing the study and culturing the cells, and was responsible for revising the statistical analysis. YL was involved in designing the study and revising the manuscript.

\section{Ethical approval and consent to participate}

Not applicable.

\section{Patient consent for publication}

Not applicable.

\section{Competing interests}

The authors declare that they have no competing interests.

\section{References}

1. Spalton D: Posterior capsule opacification: Have we made a difference? Br J Ophthalmol 97: 1-2, 2013.

2. Schaumberg DA, Dana MR, Christen WG and Glynn RJ: A systematic overview of the incidence of posterior capsule opacification. Ophthalmology 105: 1213-1221, 1998.

3. Apple DJ, Escobar-Gomez M, Zaugg B, Kleinmann G and Borkenstein AF: Modern cataract surgery: Unfinished business and unanswered questions. Surv Ophthalmol 56 (6 Suppl): S3-S53, 2011.

4. Mamuya FA, Wang Y, Roop VH, Scheiblin DA, Zajac JC and Duncan MK: The roles of alphaV integrins in lens EMT and posterior capsular opacification. J Cell Mol Med 18: 656-670, 2014.

5. Wormstone IM and Eldred JA: Experimental models for posterior capsule opacification research. Exp Eye Res 142: 2-12, 2016.

6. Zeisberg M and Neilson EG: Biomarkers for epithelial-mesenchymal transitions. J Clin Invest 119: 1429-1437, 2009.

7. Kalluri R and Weinberg RA: The basics of epithelial-mesenchymal transition. J Clin Invest 119: 1420-1428, 2009.
8. van Roy F and Berx G: The cell-cell adhesion molecule E-cadherin. Cell Mol Life Sci 65: 3756-3788, 2008.

9. Takeichi M: Cadherin cell adhesion receptors as a morphogenetic regulator. Science 251: 1451-1455, 1991.

10. Wijnhoven BP, Dinjens WN and Pignatelli M: E-cadherin-catenin cell-cell adhesion complex and human cancer. Br J Surg 87: 992-1005, 2000.

11. Lovicu FJ, Shin EH and McAvoy JW: Fibrosis in the lens. Sprouty regulation of TGF $\beta$-signaling prevents lens EMT leading to cataract. Exp Eye Res 142: 92-101, 2016.

12. Erler P and Monaghan JR: The link between injury-induced stress and regenerative phenomena: A cellular and genetic synopsis. Biochim Biophys Acta 1849: 454-461, 2015.

13. El-Hussuna A, Qvist N, Zangenberg MS, Langkilde A, Siersma V, Hjort $S$ and Gögenur I: No effect of anti-TNF-a agents on the surgical stress response in patients with inflammatory bowel disease undergoing bowel resections: A prospective multi-center pilot study. BMC Surg 18: 91, 2018.

14. Nakka VP, Prakash-Babu P and Vemuganti R: Crosstalk between endoplasmic reticulum stress, oxidative stress, and autophagy: Potential therapeutic targets for acute CNS injuries. Mol Neurobiol 53: 532-544, 2016.

15. Shen X, Xue Y, Si Y, Wang Q, Wang Z, Yuan J and Zhang X: The unfolded protein response potentiates epithelial-to-mesenchymal transition (EMT) of gastric cancer cells under severe hypoxic conditions. Med Oncol 32: 447, 2015.

16. Shin HS, Ryu ES, Oh ES and Kang DH: Endoplasmic reticulum stress as a novel target to ameliorate epithelial-to-mesenchymal transition and apoptosis of human peritoneal mesothelial cells. Lab Invest 95: 1157-1173, 2015.

17. Walter P and Ron D: The unfolded protein response: From stress pathway to homeostatic regulation. Science 334: 1081-1086, 2011.

18. Lindholm D, Korhonen L, Eriksson O and Kõks S: Recent Insights into the role of unfolded protein response in ER stress in health and disease. Front Cell Dev Biol 5: 48, 2017.

19. Yang J, Zhou S, Gu J, Wang Y, Guo M and Liu Y: Differences in unfolded protein response pathway activation in the lenses of three types of cataracts. PLoS One 10: e0130705, 2015.

20. Puls TJ, Tan X, Whittington CF and Voytik-Harbin SL: 3D collagen fibrillar microstructure guides pancreatic cancer cell phenotype and serves as a critical design parameter for phenotypic models of EMT. PLoS One 12: e0188870, 2017.

21. Cano A, Pérez-Moreno MA, Rodrigo I, Locascio A, Blanco MJ, del Barrio MG, Portillo F and Nieto MA: The transcription factor snail controls epithelial-mesenchymal transitions by repressing E-cadherin expression. Nat Cell Biol 2: 76-83, 2000.

22. Zhong Q, Zhou B, Ann DK, Minoo P, Liu Y, Banfalvi A, Krishnaveni MS, Dubourd M, Demaio L, Willis BC, et al: Role of endoplasmic reticulum stress in epithelial-mesenchymal transition of alveolar epithelial cells: Effects of misfolded surfactant protein. Am J Respir Cell Mol Biol 45: 498-509, 2011.

23. Pallet N: New insights on stress-induced epithelial phenotypic changes. Nephrol Dial Transplant 27: 483-485, 2012.

24. Tanjore H, Cheng DS, Degryse AL, Zoz DF, Abdolrasulnia R, Lawson WE and Blackwell TS: Alveolar epithelial cells undergo epithelial-to-mesenchymal transition in response to endoplasmic reticulum stress. J Biol Chem 286: 30972-30980, 2011.

25. Lawson WE, Crossno PF, Polosukhin VV, Roldan J, Cheng DS, Lane KB, Blackwell TR, Xu C, Markin C, Ware LB, et al: Endoplasmic reticulum stress in alveolar epithelial cells is prominent in IPF: Association with altered surfactant protein processing and herpesvirus infection. Am J Physiol Lung Cell Mol Physiol 294: L1119-L1126, 2008.

26. Shah PP, Dupre TV, Siskind LJ and Beverly LJ: Common cytotoxic chemotherapeutics induce epithelial-mesenchymal transition (EMT) downstream of ER stress. Oncotarget 8: 22625-22639, 2017.

27. de Iongh RU, Wederell E, Lovicu FJ and McAvoy JW: Transforming growth factor-beta-induced epithelial-mesenchymal transition in the lens: A model for cataract formation. Cells Tissues Organs 179: 43-55, 2005

28. Hales AM, Schulz MW, Chamberlain CG and McAvoy JW: TGF-beta 1 induces lens cells to accumulate alpha-smooth muscle actin, a marker for subcapsular cataracts. Curr Eye Res 13: 885-890, 1994. International (CC BY-NC-ND 4.0) License. 